\title{
Análisis de sistemas de potencia usando Scilab
}

\author{
Alexander Céspedes-F.*, Carlos A. Lozano, *\$ \\ * Grupo de Investigación en Alta Tension GRALTA - Universidad del Valle, Cali, Colombia \\ §e-mail: clozano@univalle.edu.co
}

(Recibido: Octubre 5 de 2005 - Aceptado: Enero 16 de 2006)

\begin{abstract}
Resumen
Cada día es más importante el uso del computador en el análisis de los problemas en ingeniería eléctrica; es igualmente importante, estimular la aplicación de herramientas de computador de uso general (plataformas de desarrollo matemático) en la implementación de soluciones de ingeniería. En este artículo se presenta una caja de herramientas (toolbox) desarrollada en una plataforma de desarrollo matemático de distribución libre llamada Scilab, con el propósito de servir de base para el análisis de sistemas de potencia. Así mismo, se hace una descripción general de Scilab y los algoritmos de análisis de sistemas de potencia a implementar en la caja de herramientas, que permite realizar análisis de sistemas de potencia en estado estable incluyendo análisis de flujo de carga, cortocircuito, estabilidad y despacho económico.
\end{abstract}

Palabras Clave: Flujo de carga, jacobiano, estabilidad transitoria en sistemas de potencia, corrientes de cortocircuito.

\begin{abstract}
Every day it is even more important the use of computers for the analysis of electrical engineering problems; it is also important to encourage the application of general use computer tools (mathematical development platforms) in the implementation of engineering solutions. In this paper, a toolbox developed in a free distribution mathematical platform, called Scilab, is presented, which is useful for power system analysis. At the same time, a general description of Scilab and the implemented algorithms in the toolbox is provided. The implemented toolbox allows to carry out power systems analysis in steady-state including power flow, short-circuit, stability and economic dispatch.
\end{abstract}

Keywords: Load flow, Jacobian matrices, power system transient stability, short-circuit currents.

\section{Introducción}

El análisis de sistemas de potencia existe desde el momento en que se instalaron los primeros sistemas de transmisión y distribución de energía a finales del siglo XIX. A medida que estos se desarrollaban, se requirió también avanzar en los métodos de estudio y análisis, abriendo la posibilidad de usar el computador como herramienta para la solución de los problemas operativos.

Hoy en día existen gran cantidad de programas de análisis de sistemas de potencia y el computador es una pieza clave en labores de planeamiento, diseño y análisis de operación. El tamaño y complejidad de los sistemas de potencia actuales requiere de programas computacionales eficientes, capaces de operar planteamientos de ecuaciones matemáticas 
complejas y manejo matricial óptimo. La aplicación de una caja de herramientas bajo el entorno de Scilab trata de aprovechar las ventajas de análisis matemático que éste contiene y su versatilidad al momento de realizar operaciones matriciales.

\section{El entorno de Scilab}

Scilab es un software de cálculo científico orientado a la computación numérica. Posee una extraordinaria versatilidad y capacidad para resolver problemas de matemática aplicada, física, ingeniería, procesamiento de señales y otras. $\mathrm{Su}$ base la constituye un sofisticado intérprete formado por cientos de rutinas de cálculo matricial, análisis numérico y visualización gráfica. Fue desarrollado en el INRIA, Institut National de Recherche en Informatique et Automatique, con la colaboración de la escuela de ingenieros ENPC, Ecole Nationale de Ponts et Chaussées.

La conjugación de los algoritmos implementados para el análisis de problemas comunes en sistemas de potencia tales como flujo de carga, análisis de estabilidad, despacho económico, análisis de cortocircuito entre otros, y las ventajas potenciales de Scilab permiten la implementación de programas que componen el toolbox, aprovechando las herramientas de análisis numérico provistas por esta plataforma.

\section{Funcionalidad de Scilab en sistemas de potencia}

Cada una de las funciones que han sido implementadas para el análisis de sistemas de potencia emplea de manera directa o indirecta las herramientas matemáticas de Scilab. Dichas funciones incluidas en el toolbox se describen a continuación.

\section{A) Análisis de Flujo de Carga}

Los estudios de flujos de carga son de gran importancia en la planeación y diseño de la expansión futura de los sistemas de potencia, así como también en la determinación de las mejores condiciones de operación de la red. La información principalmente que se obtiene de un estudio de flujos de cargas es la magnitud y el ángulo de fase de la tensión en cada barra, al igual que las potencias activa y reactiva en cada línea.

El análisis de flujo de carga incluido en el toolbox implementa dos métodos: el método de Gauss-Seidel y el método de Newton-Raphson. Ambos métodos se encargan de solucionar sistemas de ecuaciones que surgen al momento de plantear el balance de potencia en cada una de las barras (nodos) del sistema. De lo anterior tenemos:

$S_{i}=V_{i} I_{i}^{*}$

Donde $\mathrm{S}_{\mathrm{i}}$ : potencia compleja, $\mathrm{V}_{\mathrm{i}}$ : tensión en la barra $\mathrm{i}$, $\mathrm{I}_{\mathrm{i}}$ : corriente inyectada a la barra $\mathrm{i}$.

Basados en la ecuación anterior, los algoritmos de flujo de carga buscan la solución del sistema. En el caso del método de Gauss-Seidel, se utiliza el modo rectangular de las ecuaciones de red, asignando valores iniciales de magnitud y ángulo de tensión al igual que valores iniciales de potencia. Luego, se procede a calcular el nuevo valor de tensión de la barra $i$ en función de los valores de tensión de las barras restantes del sistema.

El método de Newton-Raphson utiliza la forma polar de las ecuaciones de la red de potencia, teniendo como incógnitas la magnitud y el ángulo de la tensión de cada barra, basándose en la multiplicación de la matriz de potencias calculadas y la matriz Jacobiana. El sistema de ecuaciones corresponde a:

Si se multiplica el vector de potencia activa y reactiva por el inverso de la matriz Jacobiana, se obtendrán los nuevos ángulos y magnitudes corregidas de tensión del sistema.

$$
\begin{aligned}
& \left|\frac{\Delta P_{i}}{\Delta Q_{i}}\right|=\left|\begin{array}{ll}
J_{11} & J_{12} \\
J_{21} & J_{22}
\end{array}\right| \bullet\left|\frac{\Delta \delta}{\Delta V}\right| \\
& \text { error }=\text { Jacobiano } \bullet \text { corrección }
\end{aligned}
$$

Los algoritmos anteriores finalizan cuando se alcanza una condición mínima de error entre las tensiones calculadas para cada iteración, para el caso del método de Gauss-Seidel, y de la condición de error calculado entre las potencias, calculadas para el caso del método de Newton-Raphson. 
Aplicación en Scilab: En el caso del método de Gauss-Seidel, Scilab trabaja con variables complejas y matrices de gran tamaño (debido al número de barras de la red), ejecutando funciones propias del programa como es el caso del intercambio filacolumna o la multiplicación de toda una matriz por un escalar.

El método de Newton-Raphson contiene un paso crítico en su algoritmo debido a la inversión de la matriz Jacobiana para el cálculo de los cambios de potencia y tensión. La ventaja que ofrece Scilab es que posee una función de inversión de matrices (inv ()) optimizada, la cual ahorra tiempo considerable. Newton-Raphson también exige el trabajo con números complejos en su forma polar. En este caso, Scilab ofrece funciones que manipulan números complejos en forma polar incluyendo el cálculo de su magnitud (abs ()), del argumento del número complejo $(\arg ())$, el cálculo del conjugado (conj ()), entre otros.

Caso de Estudio: El ejemplo utilizado en las simulaciones de flujo de carga es un sistema IEEE de 5 barras. El diagrama unificar del sistema y sus datos se muestran a continuación:

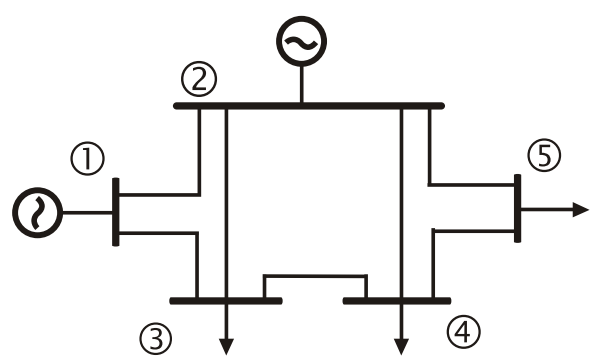

Figura 1. Diagrama Unifilar del Sistema IEEE de 5 Barras utilizado en la simulación.

Tabla 1. Datos de las líneas del Sistema IEEE de 5 Barras utilizado en la simulación.

\begin{tabular}{|c|c|c|c|c|}
\hline \multicolumn{2}{|c|}{ Barra } & \multirow{2}{*}{$\begin{array}{c}\text { Resistencia } \\
\text { [p.u] }\end{array}$} & \multirow{2}{*}{$\begin{array}{c}\text { Reactancia } \\
\text { [p.u] }\end{array}$} & \multirow{2}{*}{$\begin{array}{l}\text { Admitancia } \\
\text { Shunt [p.u] }\end{array}$} \\
\hline Partida & Llegada & & & \\
\hline 1 & 2 & 0,02 & 0,06 & 0,030 \\
\hline 1 & 3 & 0,08 & 0,24 & 0,025 \\
\hline 2 & 3 & 0,06 & 0,18 & 0,020 \\
\hline 2 & 4 & 0,06 & 0,18 & 0,020 \\
\hline 2 & 5 & 0,04 & 0,12 & 0,015 \\
\hline 3 & 4 & 0,01 & 0,03 & 0,010 \\
\hline 4 & 5 & 0,08 & 0,24 & 0,025 \\
\hline
\end{tabular}

Tabla 2. Datos de los generadores del Sistema IEEE de 5 Barras utilizado en la simulación.

\begin{tabular}{ccccc}
\hline Barra & \multicolumn{2}{c}{$\begin{array}{c}\text { Potencia Activa } \\
{[\mathrm{p} . \mathrm{u}]}\end{array}$} & \multicolumn{2}{c}{$\begin{array}{c}\text { Potencia Reactiva } \\
{[\mathrm{p} . \mathrm{u}]}\end{array}$} \\
\hline No. & Máxima & Mínima & Máxima & Mínima \\
1 & 1 & 6,25 & 0 & 0,2 \\
2 & 1 & 6,25 & 0 & 0,2 \\
\hline
\end{tabular}

El programa arroja resultados gráficos como los indicadores de tensión vs. Barra y ángulo vs. Barra del sistema. Para la salida de datos se hace uso de los recursos de graficación de Scilab con las funciones plot () y plot $2 d$ ().
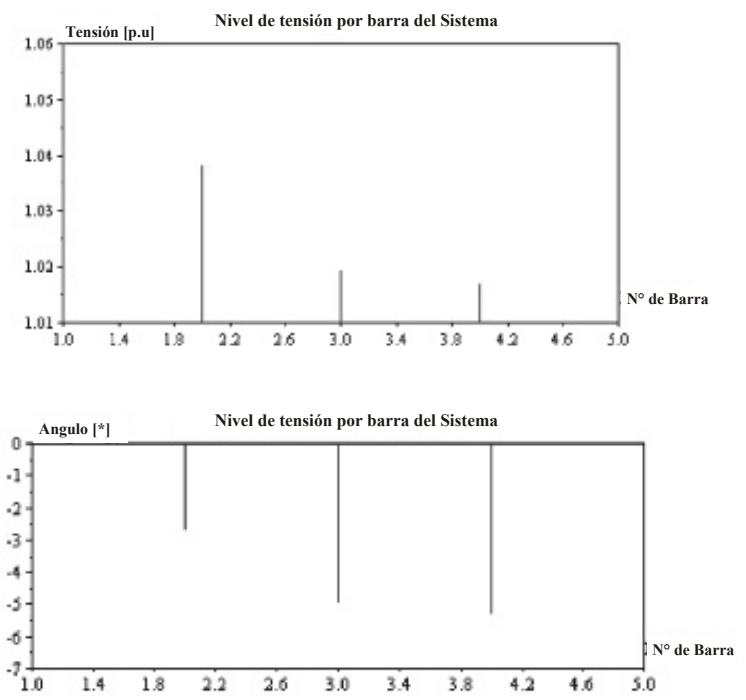

Figura 2. Gráficas Magnitud y Ángulo de tensión vs. Barra, arrojadas por el análisis de flujo de carga.

A partir de estos resultados se pueden apreciar de una manera sencilla los valores más y menos significativos de magnitud y ángulo de tensión en las barras, lo cual es ideal para establecer las violaciones en los límites establecidos en la red de potencia.

\section{B) Despacho Económico}

La operación económica de un sistema de potencia es importante para analizar el impacto de las decisiones operativas en la red. La máxima eficiencia se obtiene al minimizar el costo del kilowatt-hora, considerando la coordinación de los costos de producción en todas las plantas generadoras que operan en el sistema. El problema puede ser analizado considerando o no las 
pérdidas eléctricas en la red. El despacho económico sin pérdidas toma las ecuaciones de costos de los generadores involucrados y halla el costo incremental que minimiza los costos de generación a un determinado nivel de potencia, conduciendo a la ecuación:

$$
\lambda_{i}=\frac{d f_{i}}{d P_{g i}}=a_{i} P_{g i}+b_{i}
$$

Donde $f_{i}$ es la función de costos de cada generador, $1_{i}$ es el costo incremental de la unidad $i, P_{g i}$ es la salida de la unidad $i$, en megawatts (MW) y $a_{i}$ y $b_{i}$ son constantes propias de la ecuación de costo incremental de cada generador.

El criterio para una distribución económica de carga entre unidades generadoras, es que todas las unidades deben operar al mismo costo incremental de combustible.

El programa para el despacho económico, considerando pérdidas, se ejecuta de la misma manera pero añade el cálculo de la potencia consumida en las líneas (pérdidas activas de red) basándose en la matriz de coeficientes $B$ del sistema (coeficientes de pérdidas) que caracterizan las pérdidas de transmisión del mismo.

Aplicación en Scilab: Se consideran dos posibilidades de análisis: la primera conocida como el despacho económico sin pérdidas y cuyo requerimiento básico es la ejecución correcta de las sumatorias de las potencias para el cálculo del costo incremental de los generadores; la segunda posibilidad, es el despacho económico con pérdidas, el cual incluye un factor adicional, los coeficientes $B$, $\mathrm{y}$ de nuevo un manejo matricial de las ecuaciones del sistema.

Caso de Estudio: En el caso de despacho económico se realizó el análisis utilizando los datos de costos de los generadores del sistema IEEE de 5 Barras (Fig. 1) como se muestra a continuación:

Tabla 3. Datos económicos de los generadores del Sistema IEEE de 5 Barras utilizado en la simulación.

\begin{tabular}{cccc}
\hline Barra & \multicolumn{3}{c}{ Coeficientes de Costo Incremental } \\
\hline No. & A & B & C \\
1 & 0,0080 & 8,0 & 0,0 \\
2 & 0,0096 & 6,4 & 0,0 \\
\hline
\end{tabular}

Para la evaluación del sistema considerando pérdidas se calculó la siguiente matriz de coeficientes de pérdidas basados en los datos de las líneas del sistema (Tabla 1).

$$
\left[\begin{array}{ccc}
8,383183 & -0,049448 & 0,375082 \\
-0,049448 & 5,963568 & 0,194971 \\
0,375082 & 0,194971 & 0,090121
\end{array}\right]
$$

Figura 3. Matriz de Coeficientes B del Sistema IEEE de 5 Barras utilizado en la simulación

Ambos programas arrojaron los siguientes resultados:

Tabla 4. Resultados del despacho económico sin péndidas y considerando pérdidas para un sistema de dos generadores.

\begin{tabular}{cccccccc}
\hline $\begin{array}{c}\text { Potencia } \\
\text { de } \\
\text { demanda } \\
{[\mathrm{MW}]}\end{array}$ & $\begin{array}{c}\text { Costo } \\
\text { incremen } \\
\text { sin pérdidas }\end{array}$ & $\begin{array}{c}\text { Costo } \\
\text { incremen } \\
\text { con pérdidas }\end{array}$ & $\begin{array}{c}\text { Generac. } \\
\text { sin } \\
\text { pérdidas [MW] }\end{array}$ & $\begin{array}{c}\text { Generac. con } \\
\text { pérdidas [MW] }\end{array}$ & $\begin{array}{c}\text { Pérdidas } \\
\text { calculadas } \\
\text { [MW] }\end{array}$ \\
\cline { 4 - 6 } & & & $\mathrm{P}_{\mathrm{G} 1}$ & $\mathrm{P}_{\mathrm{G} 2}$ & $\mathrm{P}_{\mathrm{G} 1}$ & $\mathrm{P}_{\mathrm{G} 2}$ & \\
\hline 350 & 8.80 & 9.03 & 100 & 250 & 108 & 246 & 4.64 \\
400 & 9.01 & 9.29 & 127 & 272 & 135 & 270 & 5.93 \\
500 & 9.45 & 9.83 & 181 & 318 & 190 & 319 & 9.12 \\
800 & 10.76 & 11.53 & 345 & 454 & 355 & 468 & 23.60 \\
950 & 11.41 & 12.43 & 427 & 522 & 439 & 544 & 33.74 \\
1150 & 12.29 & 13.68 & 536 & 613 & 552 & 648 & 50.42 \\
\hline
\end{tabular}

\section{C) Estudio de Estabilidad}

El estado de un sistema eléctrico de potencia puede ser clasificado como estable o inestable. El punto crítico de estabilidad es cualquier condición para la cual un pequeño cambio en la operación de los elementos de red causa inestabilidad. La naturaleza de una respuesta inestable dependerá de las características del sistema y de las condiciones de operación. Aunque en general cada variable del sistema está involucrada, en casos particulares la inestabilidad puede manifestarse como la pérdida del sincronismo de una o más unidades de generación (también llamado inestabilidad de ángulo) o por la caída incontrolable de tensión del sistema en una fracción importante de la red (también llamado inestabilidad de tensión). En cualquier caso, todas las máquinas sincrónicas interconectadas deben permanecer en sincronismo si el sistema es estable, es decir, operando a la misma velocidad.

Durante la condición de estado transitorio siguiente a un disturbio, la suma de potencias no es cero, generando una aceleración o desaceleración dependiendo de si el parámetro torque de la máquina 
generadora es positivo o negativo. Esto da origen a un desplazamiento angular q, el cual puede ser calculado mediante la siguiente ecuación:

$\theta=\omega_{s} t+\delta$

Donde $\omega_{s}$ es la velocidad sincrónica y $\delta$ es el desplazamiento angular del rotor. Si se analiza un sistema con más de un generador se tendrán que resolver dos sistemas de ecuaciones diferenciales de primer orden para obtener los cambios en el ángulo de tensión interno $\delta_{i}$ y la velocidad de la máquina $\omega_{i}$. De esta manera, para un sistema con $m$ máquinas es necesario resolver $2 m$ ecuaciones diferenciales simultáneas. Esas ecuaciones son:

$$
\begin{aligned}
& \frac{d \delta_{i}}{d t}=\omega_{i(t)}-2 \pi f \\
& \frac{d \omega_{i}}{d t}=\frac{\pi f}{H_{i}}\left(P_{m i}-P_{e i(t)}\right) i=1,2, \ldots, m
\end{aligned}
$$

Existen varios métodos para solucionar el sistema de ecuaciones resultante al aplicar la ecuación (4) en el estudio de estabilidad, entre los que se pueden citar los siguientes:

Método de Euler modificado: En este método, la estimación inicial de los ángulos de la tensión interna y las velocidades de la máquina en un tiempo $t+\ddot{A} t$ son obtenidas de:

$\delta_{i(t+\Delta t)}^{(0)}=\delta_{i(t)}^{(1)}+\left.\frac{d \delta_{i}}{d t}\right|_{(t)} \Delta t$

$\omega_{i(t+\Delta t)}^{(0)}=\omega_{i(t)}^{(1)}+\left.\frac{d \omega_{i}}{d t}\right|_{(t)} \Delta t \quad i=1,2, \ldots, m$

Donde las derivadas son evaluadas de las ecuaciones (4), cuando $t=0$. La potencia de la máquina $P_{e i(\theta)}$ es obtenida al solucionar la red en el instante después de que el disturbio ocurre.

Método de Runge-Kutta de cuarto orden: En este método, se hacen cuatro estimaciones de cambios en el ángulo del rotor y velocidad de la máquina en cada intervalo. Su determinación final se realiza como sigue:

$$
\begin{aligned}
& \Delta \delta_{i(t+\Delta t)}=\frac{1}{6}\left(k_{1 i}+2 k_{2 i}+2 k_{3 i}+k_{4 i}\right) \\
& \Delta \omega_{i(t+\Delta t)}=\frac{1}{6}\left(l_{1 i}+2 l_{2 i}+2 l_{3 i}+l_{4 i}\right)
\end{aligned}
$$

En ambas expresiones, $k$ y $l$ caracterizan los cambios en $\delta$ y $\omega$ respectivamente, obtenidos a través de las derivadas de la ecuación (5) en puntos específicos.

Método Trapezoidal: En este método de cálculo el cambio en la posición angular del rotor durante un corto intervalo de tiempo se calcula bajo las siguientes suposiciones:

1.- La potencia de aceleración $P_{a}$ calculada al principio de un intervalo, es constante desde la mitad del intervalo que le precede hasta la mitad del intervalo considerado.

2.- A través de cualquier intervalo, la velocidad angular es constante e igual al valor calculado para la mitad del intervalo.

La ecuación que rige este tipo de procedimiento viene dada por:

$\Delta \delta_{n}=\delta_{n-1}+k P_{a, n-1}$

donde: $\quad k=\frac{180 f}{H}(\Delta t)^{2}$

En la ecuación (8) el término $\mathrm{D} t$ es el intervalo de tiempo escogido para realizar la aproximación de la curva de oscilación para cada iteración; de nuevo, $H$ es la constante de inercia y $f$ es la frecuencia del sistema.

Aplicación en Scilab: El análisis de estabilidad usando el método de Euler modificado, obliga a implementar funciones adicionales y utilizar funciones trigonométricas predeterminadas en Scilab tales como $\sin (), \cos (), \operatorname{atan}()$. En el análisis de estabilidad por medio del método de Runge-Kutta de cuarto orden, también se emplean las funciones a las que se recurre en Euler modificado con un manejo matricial adecuado. 
Caso de Estudio: Así como en los casos anteriores, se parte de un sistema IEEE de 5 barras. Los datos de estabilidad usados para validar el programa se muestran a continuación.

El estudio de estabilidad validado a través de cualquiera de los tres métodos descritos anteriormente arroja los siguientes resultados:

Tabla 5. Datos de estabilidad de los generadores del sistema IEEE de 5 barras.

\begin{tabular}{ccc}
\hline Barra & Constante & Reactancia \\
\hline No. & de Inercia & Subtransitoria \\
1 & 50 & 0,25 \\
2 & 1 & 1,5 \\
\hline
\end{tabular}
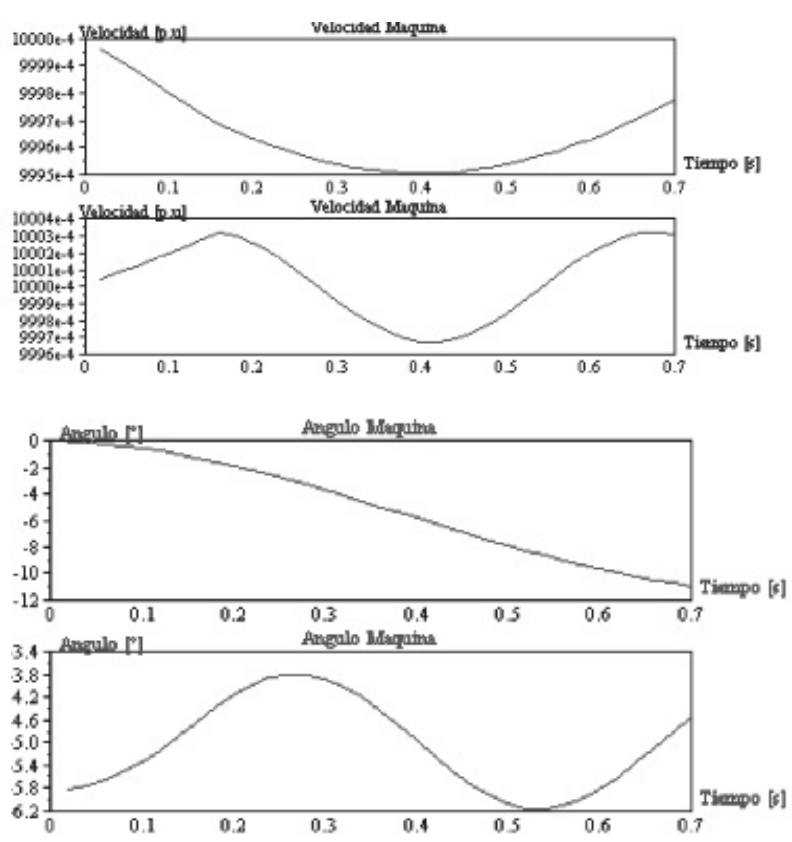

Figura 4. Resultados de la simulación de estabilidad (Velocidad y Angulo vs. Tiempo).

En las gráficas superiores se observa el comportamiento del generador 1 y en la inferior el comportamiento del generador 2. Como se puede apreciar en la figura anterior, el toolbox permite visualizar el comportamiento de todos y cada uno de los generadores involucrados en el caso de estudio, permitiendo establecer las fallas más críticas para el sistema.

\section{D) Análisis de Cortocircuito}

Un cortocircuito en cualquier parte de un sistema de potencia causa por lo general un incremento notable en la corriente. Existen muchas medidas preventivas que pueden ser adoptadas para tratar los cortocircuitos en un sistema, incluyendo los dispositivos de protección. La mayoría de los programas de análisis de cortocircuito tratan de determinar las corrientes y tensiones en diferentes puntos del sistema, correspondiente a diferentes tipos de fallas, de tal manera que los esquemas de protección e interruptores pueden ser seleccionados para rescatar el sistema de una condición anormal en cuestión de milisegundos.

La falla puede ser del tipo serie involucrando conductores rotos, fusibles quemados, etc.; o del tipo shunt o derivación incluyendo una o mas fases a tierra. Respecto a este tipo de fallas shunt se tienen varios esquemas: línea-tierra, línea-línea y doble línea-tierra, los cuales requieren para su análisis la definición de redes de secuencia. En este trabajo se implementaron rutinas tendientes a simular las fallas en derivación o shunt; las fallas en serie se considerarán en una versión futura.

Aplicación en Scilab: Para realizar un análisis de cortocircuito es necesario contar con matrices que almacenen las diferentes redes de secuencia que se pueden formar en el sistema de potencia estudiado, además de incluir algunas operaciones con complejos, necesarias para el cálculo de las tensiones y corrientes de cortocircuito.

Caso de Estudio: En el estudio de cortocircuito se escogió un sistema basado en una red típica, la cual se muestra en el siguiente diagrama unificar, simulando una falla en la barra No. 2 del sistema.

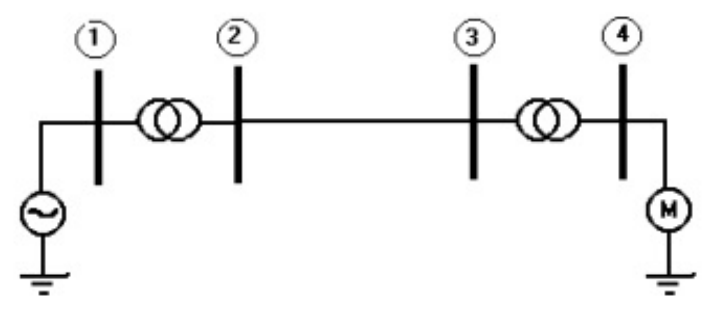

Figura 5. Diagrama Unifilar del Sistema de Potencia utilizado para la simulación de cortocircuito.

La red de secuencia cero resultante del sistema se muestra a continuación: 
Tabla 6. Datos de la red de secuencia cero del sistema usado para la simulación de cortocircuito.

\begin{tabular}{ccccc}
\hline \multicolumn{2}{c}{ Barra } & $\begin{array}{c}\text { Resistencia } \\
\text { Partida }\end{array}$ & $\begin{array}{c}\text { Reactancia } \\
\text { [p.u] }\end{array}$ & $\begin{array}{c}\text { Admitancia } \\
\text { Shunt [p.u] }\end{array}$ \\
\hline 1 & 0 & 0,00 & 0,19 & 0,00 \\
1 & 2 & 0,00 & 0,08 & 0,00 \\
2 & 3 & 0,00 & 0,50 & 0,00 \\
3 & 4 & 0,00 & 0,08 & 0,00 \\
4 & 0 & 0,00 & 0,19 & 0,00 \\
\hline
\end{tabular}

Los resultados arrojados por el programa de cortocircuito se muestran a continuación:

Tabla 7. Resultados de la simulación de cortocincuito para diferentes tipos de falla.

\begin{tabular}{cccc}
\hline $\begin{array}{c}\text { Tipo de Falla } \\
\text { Simulad a }\end{array}$ & $\begin{array}{c}\text { Tensión de } \\
\text { prefalla [p.u] }\end{array}$ & $\begin{array}{c}\text { Impedancia de } \\
\text { falla [p.u] }\end{array}$ & $\begin{array}{c}\text { Corriente secuencia } \\
\text { positiva [p.u] }\end{array}$ \\
\hline Trifás ica & 1.0 & $0.3+\mathrm{j} 5$ & 0.500 \\
Monofásica a tierra & 1.0 & $0.3+\mathrm{j} 5$ & 0.061 \\
Bifásica & 1.0 & ------ & 0.250 \\
Bifásica a tierra & 1.0 & $0.3+\mathrm{j} 5$ & 0.255 \\
\hline
\end{tabular}

Evidentemente el análisis de cortocircuito permite establecer el tipo de falla más severa para el sistema.

\section{Conclusiones}

En el desarrollo de este trabajo se pudo establecer la aplicabilidad de Scilab como plataforma matemática general para el análisis de sistemas de potencia. El paquete desarrollado utiliza las rutinas matemáticas de Scilab para la realización de cálculos matriciales de los diferentes módulos de análisis de sistemas de potencia y las aplicaciones gráficas del mismo.

Además, se comprobó la versatilidad de Scilab al momento de aplicar algoritmos complejos y de alta densidad matemática y su capacidad para el manejo de operaciones de alto grado de dificultad. En este sentido, las limitaciones de simulación del toolbox son fundamentalmente las que tiene Scilab, es decir, el número de nodos y elementos de red a simular están limitados por los algoritmos propios de la plataforma matemática.

A través de los resultados arrojados por el toolbox se puede caracterizar un sistema de potencia desde distintas ópticas como costos de generación, comportamiento ante fallas, niveles de tensión en las barras y niveles de cortocircuito debidos a variaciones en las condiciones de carga y topología.
La herramienta desarrollada es útil en aplicaciones académicas, como herramienta de entrenamiento para los estudiantes en la aplicación de algoritmos numéricos. Es también un paquete importante de simulación para diferentes casos de análisis, que abre las puertas para futuros desarrollos de mayor cobertura y complejidad en sistemas de potencia.

La herramienta implementada (toolbox) se puso a disposición de la comunidad de usuarios de Scilab y se encuentra en la sección de contribuciones de la página principal de Scilab (http://www.scilab.org).

\section{Referencias bibliográficas.}

[1] Anderson, P. M. Analysis of Faulted Power System. Iowa: The Iowa State University Press. $1981.513 \mathrm{p}$.

[2] Anderson, P. M. y Fouad, A. Power System Control and Stability. Nueva York: Institute of Electrical and Electronics Enginners IEEE. 1994. $465 \mathrm{p}$.

[3] Corredor, P. H. Operación Económica de Sistemas de Potencia. Medellín: Universidad Pontificia Bolivariana. 1992.250 p.

[4] Dhar, R.N. Computer Aided Power System Operation and Analysis. New York: Mc. Graw Hill, 1982.266 p.

[5] Grainger, J. y Stevenson, W. Análisis de Sistemas de Potencia. México: Mc. Graw Hill, 1997.740 p.

[6] Introduction To Scilab. Scilab Group. Institut National de Recherche en Informatique et Automatique (INRIA). Manuales On-line. 2003.

[7] Jiménez. J, A. Scilab. Computación numérica bajo Linux y Windows (PDF). Universidad de Cádiz. España. 2001.

[8] Stagg, G., El-abiad, A. Computer Methods in Power Systems Analysis. Nueva York: Mc. Graw Hill, 1968. 427 p. 